\title{
Is there any role of glucose-6-phosphate dehydrogenase in obesity induced metabolic disorder
}

\author{
Manisha Sankhla ${ }^{1^{*}}$, Keerti Mathur ${ }^{2}$, Jai Singh Rathor ${ }^{3}$ \\ ${ }^{1}$ Department of Physiology, Mahatma Gandhi Medical College and Hospital, Jaipur (Rajasthan), India; \\ *Corresponding Author: manisha_signsky@yahoo.com \\ ${ }^{2}$ Department of Physiology, S.M.S. Medical College, Jaipur (Rajasthan), India \\ ${ }^{3}$ Medical Officer, Primary Health Center, Badhal (Rajasthan), India
}

Received 10 November 2012; revised 16 December 2012; accepted 27 December 2012

\begin{abstract}
The present study was designed to explore the possible mechanism of obesity associated metabolic syndrome. 150 subjects $(120$ men and 30 women) in the age-group of 17 - 26 years were studied. Body Mass Index and Waist-to-Hip Ratio were taken as a measure of generalized obesity and abdominal adiposity. The serum concentration of glucose-6-phosphate dehydrogenase increased with increasing levels of Body Mass Index and was found to be significant in obese subjects (Body Mass Index $\geq 30.0 \mathrm{~kg} / \mathrm{m}^{2}$ ) and more so in the obese subjects with abdominal adiposity $(p=0.002)$ as compared to normal-weight subjects. Karl Pearson coefficient of correlation revealed a significant positive correlation of glucose-6-phosphate dehydrogenase with Body Mass Index ( $r=0.499 ; p<$ 0.001 ) and malondialdehyde (a biomarker of oxidative stress) $(r=0.736 ; p<0.001)$ but inverse correlation with adiponectin $(r=-0.524 ; p$ $<0.001$ ). Thus, we conclude that increased expression of glucose-6-phosphate dehydrogenase in obese subjects (more if it is associated with abdominal adiposity) might mediate the onset of obesity associated metabolic disorders by increasing oxidative stress.
\end{abstract}

Keywords: Obesity; Abdominal Adiposity; Oxidative Stress; Glucose-6-Phosphate Dehydrogenase;

Adiponectin

\section{INTRODUCTION}

Obesity is an important predictor of health issues [1]. The increasing epidemic of obesity and metabolic disor- ders is a growing medical problem in developed and developing countries $[2,3]$. Obesity is the central and causal component in metabolic syndrome [4], but the mechanistic role of obesity has not been elucidated.

Obesity represents increase in adipose tissue mass and adipocytes produce a variety of biologically active molecules known as adipocytokines including tumor necrosis factor- $\alpha$, Interleukin- 6 , resistin, leptin and adiponectin $[5,6]$. Dysregulated production of these adipocytokines participates in the pathogenesis of obesity-associated metabolic syndrome [7]. Among these adipocytokines, adiponectin is unique adipocyte-derived hormone, as increase fatness is associated with its lower concentration [8]. However, the mechanism by which fat accumulation leads to such dysregulation of adiponectin has not been elucidated.

Moreover, obesity is associated with an increased production of reactive oxygen species (ROS) [9]. Despite this, question of how obesity produce oxidative stress remains to be elucidated. One of the major sources of cellular reactive oxygen species is mitochondria [10], and another source are pro-oxidative enzymes such as NADPH oxidase (Nicotinamide Adenine Dinucleotide Phosphate) and inducible nitric oxide synthase (iNOS) and their abnormal increase stimulates oxidative stress $[11,12]$.

A balance between the generation of ROS and the ROS-scavenging anti-oxidant system, regulated by the intracellular $\mathrm{NAD}(\mathrm{P}) \mathrm{H}$-to-NAD $(\mathrm{P})^{+}$ratio, determines oxidative stress. Interestingly, NADPH is the central component of both pro and anti-oxidant processes. Therefore, the intracellular $\mathrm{NAD}(\mathrm{P}) \mathrm{H}$-to- $\mathrm{NAD}(\mathrm{P})^{+}$ratio appears to be essential for the determination of cellular redox potential. The enzymatic activities of NADPH oxidase and iNOS (inducible nitric oxide synthase), the major enzymes involved in oxidative stress were elevated in obesity, moreover, the level of antioxidative 
enzymes decreases [12]. Thus, the production of ROS increases in obesity, results into reduction in NAD $(\mathrm{P}) \mathrm{H}-$ to- $\mathrm{NAD}(\mathrm{P})^{+}$ratio, which leads to oxidative stress in obesity.

NADPH is produced by several enzymes including Malic Enzyme and the first two enzymes of Hexose Monophosphate pathway (HMP), glucose-6-phosphate dehydrogenase (G6PD) and 6-phosphogluconate dehydrogenase [13]. The tissues such as liver, adipose tissue, adrenal gland, erythrocytes, testes and lactating mammary gland, are highly active in HMP shunt. Most of these tissues are involved in the biosynthesis of fatty acids and steroids, which are dependent on the supply of NADPH, so it is plausible to speculate that NADPH producing enzymes might be associated with lipid metabolism disorders such as hyperlipidemia and lipid toxicity in metabolic diseases, including obesity and diabetes.

Although, isocitrate dehydrogenase also generates $\mathrm{NADPH}$, its major function is irrelevant to lipogenesis $[13,14]$. Among the four NADPH producing enzymes G6PD, is the first and rate limiting enzyme of HMP and is a major intracellular source of NADPH generation, highly conserved in most mammalian species [15]. Thus, G6PD plays a key role and is a crucial enzyme in the maintenance of cellular redox potential and cell survival via production of NADPH.

With this background in mind, the present study was designed to investigate the association of G6PD with obesity and its relation with obesity induced oxidative stress and adipocytokine-adiponectin.

\section{SUBJECTS AND METHODS}

Present study was conducted in the upgraded Department of Physiology, S.M.S. Medical College, Jaipur (Rajasthan) on 150 subjects (120 men and 30 women) in the age-group of 17 - 26 years, recruited from various camps organized at Jaipur, through verbal and public advertisements.

All the subjects were categorized into two groups:

Group-I: Normal weight subjects (Body Mass Index = $18.5-24.9 \mathrm{~kg} / \mathrm{m}^{2}$ )

Group-II: Obese subjects (Body Mass Index $\geq 25.0$ $\mathrm{kg} / \mathrm{m}^{2}$ )

This group was further divided into two classes:

Class-1: Generalized obesity without abdominal adiposity.

Class-2: Generalized obesity with abdominal adiposity.

\subsection{Study Population: Inclusion Criteria}

120 subjects of Group-II (40 Class-1 and 80 Class-2) and 30 age-matched healthy control subjects (Group-I) of either sex were recruited. Group-I subjects having normal waist-to-hip ratio were included in the study. All the participants gave written informed consent before enrollment and the study was approved by the Research Board of "Rajasthan University of Health Sciences, Jaipur (Rajasthan), India".

\subsection{Exclusion Criteria}

Individuals were excluded, who reported having a doctor diagnosed illness including hypertension, diabetes, cardiovascular disease etc or any associated illness, or receiving any form of medication/therapies or under stress, smokers, alcoholic, tobacco user, based on their responses to the questionnaire.

Anthropometric measurements, clinical examination and a detailed history of each subject regarding the age, gender, family history of obesity and of any associated illness or stress was recorded. Blood Pressure, height, weight, waist and hip circumference were obtained using standardized equipment and procedures. Body Mass Index (BMI) and Waist-Hip Ratio (WHR) were taken as a measure of generalized obesity and abdominal adiposity, respectively.

\subsection{Metabolic Variables}

Blood sample of each subject was analyzed for Fasting Blood Glucose [16], lipid profile parameters-triglyceride [17], total cholesterol [18] and high density lipoprotein cholesterol [19] using standard commercially available enzymatic kits using autoanalyser while low density lipoprotein cholesterol and very low density lipoprotein cholesterol by Friedwald's Formula [20]. The RayBio ${ }^{\circledR}$ Human Adiponectin (Acrp30) ELISA (Enzyme-Linked Immunosorbent Assay) kit was used for quantitative measurement of human adiponectin in serum. Serum Malondialdehyde (MDA, used as a biomarker of oxidative stress) was estimated by Thiobarbituric acid assay method [21] by spectrophotometrically. The quantitative estimation of serum G6PD was done by Kinetic enzyme method [22] on semi-autoanalyzer. The activity of the enzymeGlucose-6-phosphate dehydrogenase was defined by measuring the speed of the absorbance increase at 340 $\mathrm{nm}$ due to the reduction of $\mathrm{NADP}^{+}$according to the following reaction:

$$
\begin{gathered}
\text { Glucose-6-phosphate }+ \text { NADP }^{+} \\
\text {G6PD } \\
\text {--------------> } \\
\text { Gluconate-6-phosphate + NADPH }+\mathrm{H}^{+}
\end{gathered}
$$

\subsection{Diagnostic Criteria}

The National Institutes of Health (NIH) [23] classification was used for grading BMI and sex-specific Waist- 
to-Hip Ratio quartiles as recommended by World Health Organization [24] was used for analyses of abdominal adiposity. Diabetes was diagnosed based on revised American Diagnostic Association Criteria (ADA) [25] and dyslipidemia by using National Cholesterol Education Program (NCEP) [26].

\subsection{Statistical Analysis}

Statistical analysis of data were performed using the SYSTAT (version 11.00) software package. Data were expressed as mean \pm SD. Unpaired Student's “ $t$ " test was used for analysis of data and Karl Pearson coefficient of correlation was also computed to quantify the degree and direction of association of G6PD with BMI, MDA and adiponectin. The statistical significance was set at $\mathrm{p}<$ 0.05 .

\section{RESULTS}

Table 1 showed the demographic profile of studied population. The mean age of the studied subjects was $21.98 \pm 3.08$ years. The majority of subjects had no family history of obesity and the ratio between positive and negative family history of obesity was $1: 14$. None of the subjects had diabetes (according to revised ADA criteria, mean value of fasting blood glucose $=92.86 \pm 12.23$ $\mathrm{mg} / \mathrm{dL}$ ) and hypertension (mean systolic blood pressure $=123.57 \pm 5.63 \mathrm{mmHg}$ and mean diastolic blood pressure $79.48 \pm 3.65 \mathrm{mmHg}$ ), or dyslipidemia (as per NCEP guidelines).

Out of total 150 subjects, $20 \%$ of the subjects had normal weight $\left(\mathrm{BMI}=18.5-24.9 \mathrm{~kg} / \mathrm{m}^{2}\right)$ as per the $\mathrm{NIH}$

Table 1. Demographic profile of studied population.

\begin{tabular}{cc}
\hline Variables & MEAN \pm SD \\
\hline Age (years) & $21.98 \pm 3.08$ \\
Sex $(\mathrm{M} / \mathrm{F})$ & $120 / 30$ \\
Family History of Obesity (+/-) & $10 / 140$ \\
Body Mass Index (kg/m²) & $27.48 \pm 4.00$ \\
Waist-to-Hip Ratio & $0.93 \pm 0.08$ \\
Systolic Blood Pressure (mmHg) & $123.57 \pm 5.63$ \\
Diastolic Blood Pressure (mmHg) & $79.48 \pm 3.65$ \\
Fasting Blood Glucose (mg/dl) & $92.86 \pm 12.23$ \\
Total Cholesterol (mg/dl) & $179.17 \pm 21.38$ \\
LDL-Cholesterol (mg/dl) & $101.23 \pm 21.93$ \\
HDL-Cholesterol (mg/dl) & $52.86 \pm 5.11$ \\
VLDL-Cholesterol (mg/dl) & $25.07 \pm 4.21$ \\
Triglyceride (mg/dl) & $125.38 \pm 21.05$ \\
\hline
\end{tabular}

classification of BMI and rests (80\%) were overweight/ obese. Maximum (54.67\%) of the subjects were overweight $\left(\mathrm{BMI}=25.0-29.9 \mathrm{~kg} / \mathrm{m}^{2}\right)$. As per the present study groups, out of total 120 obese subjects, $66.67 \%$ of the subjects had abdominal adiposity whereas remaining $33.33 \%$ had normal WHR, depicting a high association of abdominal adiposity with generalized obesity.

Figure 1 demonstrate that on comparison with normal-weight subjects the concentration of G6PD increased with increasing levels of BMI (as per the NIH classification) and the level of significance also increased statistically (obese class-I p $=0.01$; obese class-II p = 0.001 ) except in overweight subjects.

According to present study groups (Figure 2), on comparison with normal-weight subjects, a statistically significant higher serum G6PD level was observed only in obese subjects with abdominal adiposity $(p=0.002)$ while a non-significant higher concentration of G6PD in obese subjects without abdominal adiposity.

Karl Pearson coefficient of correlation revealed a statistically significant positive correlation of G6PD with BMI $(\mathrm{r}=0.499 ; \mathrm{p}<0.001)$ and MDA $(\mathrm{r}=0.736 ; \mathrm{p}<$ $0.001)$ but inverse correlation with adiponectin $(\mathrm{r}=$ $-0.524 ; \mathrm{p}<0.001)$.

\section{DISCUSSION}

Previous research on G6PD mostly focused on erythrocytes, since G6PD deficiency in erythrocytes is a common human enzymopathy, which results from a mutation in the G6PD gene [27]. Furthermore, it was reported that G6PD deficient patients showed a decreased lipogenic rate and serum lipoprotein concentrations, implying the importance of G6PD in fatty acid synthesis [28]. Nonetheless, the functional roles of G6PD in adipocytes are poorly understood.

Furukawal et al. [12] suggested that NADPH oxidase

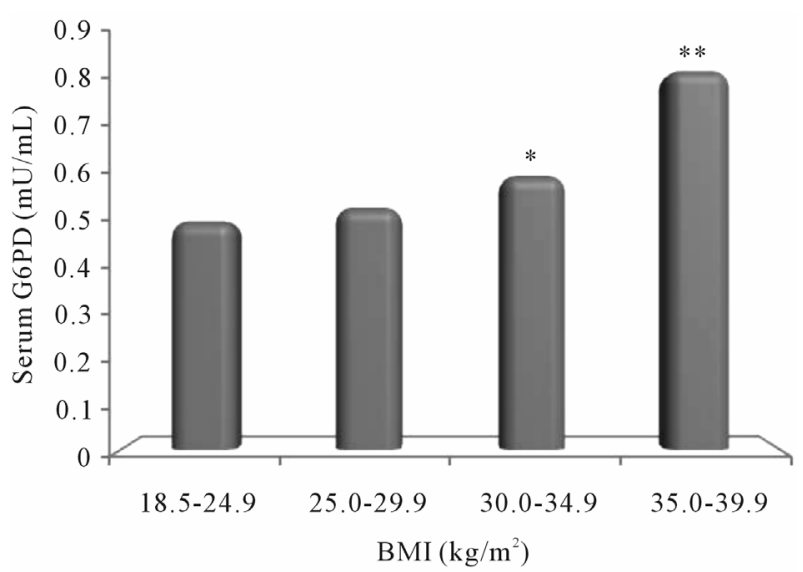

Figure 1. Relationship of Glucose-6-phosphate dehydrogenase (G6PD) with Body Mass Index (as per the NIH classification). Note : ${ }^{*}<0.05 ;{ }^{* *}<0.01$. 
Normal weight Obese without adiposity
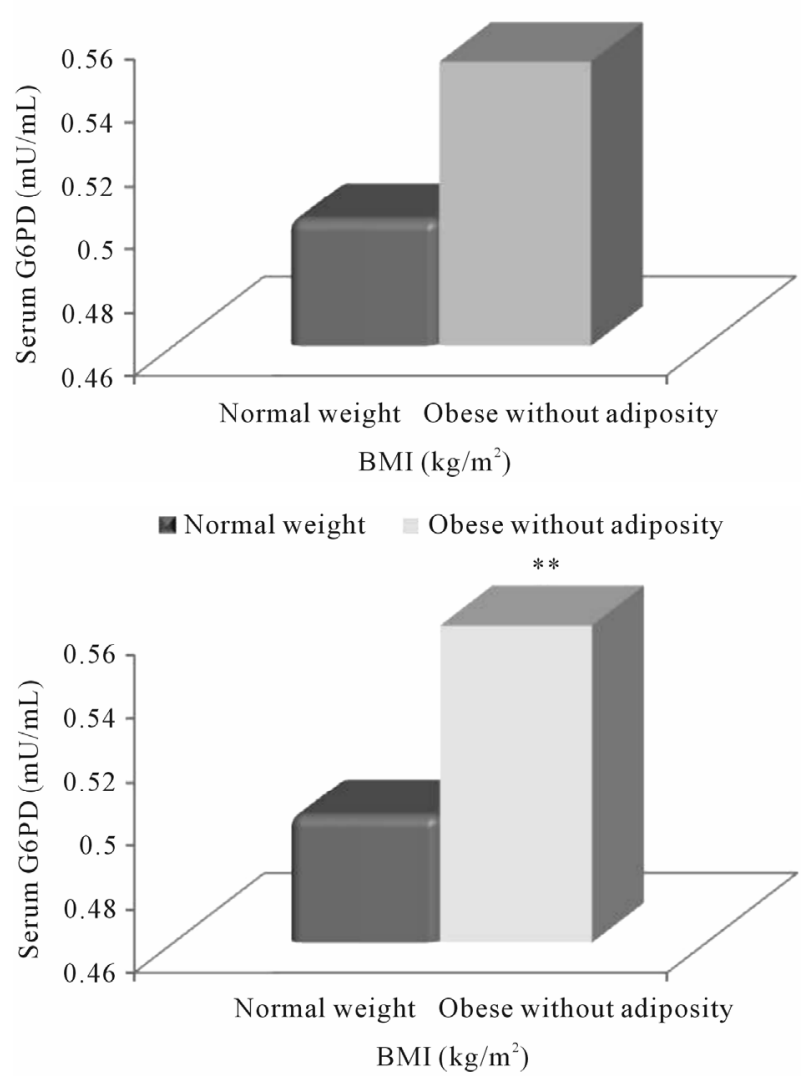

Figure 2. Association of Glucose-6-phosphate (G6PD) with obesity according to the present study groups. Note: ${ }^{* *}<0.01$.

was the major source of ROS in adipocytes and augmented NADPH oxidase seems to contribute to increase ROS production in adipose tissue in obesity, which leads to elevation of systemic oxidative stress. Later, Carvalho et al. [29] and Yasukawa et al. [30] also suggested that the enzymatic activities of NADPH oxidase and iNOS, the major enzymes involved in oxidative stress, were elevated in obesity.

In the year 2005, Park et al. [31] revealed that the elevated oxidative stress in the adipose tissue of obese animals with high levels of NADPH oxidase activity was closely associated with the increased G6PD expression. And, an aberrant increase of G6PD (one of the major NADPH-producing enzyme) in adipocytes provoked the dysregulation of lipid metabolism and adipocytokine expression, which eventually accelerate the onset of obesity induced metabolic disorders. Thus, obesity induced lipid metabolic disorders and insulin resistance, was mediated, at least in part, by overexpression of G6PD in that tissue.

To the best of our knowledge, till now there was no human study to assess the relationship of obesity and G6PD.
The present study depicts that the serum concentration of G6PD increased with increasing levels of BMI and was found to be significant in obese subjects (BMI $\geq$ $30.0 \mathrm{~kg} / \mathrm{m}^{2}$ ) as compared to normal-weight (according to NIH classification) (Figure 1), and more so in the obese subjects with abdominal adiposity $(p=0.002)$ according to the present study groups (Figure 2). Further, present analysis of data also revealed significant positive correlation of G6PD with BMI $(r=0.499 ; p<0.001)$ and MDA $(r=0.736 ; p<0.001)$ but inverse correlation with adiponectin $(\mathrm{r}=-0.524 ; \mathrm{p}<0.001)$.

Above results can be explained by the findings observed by Park et al. [32], who suggested that overexpression of G6PD in adipocyte might mediate the onset of metabolic disorders in obesity by increasing oxidative stress and inflammatory signals. Below Figure 3, demonstrate a novel role of G6PD in obesity induced metabolic disorders [32].

Moreover, it had been demonstrated in numerous studies that oxidative stress induce activation of NF- $\mathrm{KB}$ signaling and it appears to be associated with the pathogenesis of insulin resistance and type-2 diabetes [33,34].

Taken together, we propose that the G6PD expression levels in adipose tissue may be a promising indicator of obesity and its regulation may be a novel therapeutic target in treating metabolic disorders such as obesityinduced insulin resistance and chronic inflammation.

Discrepancies among the results within the subset of obese groups may be because of the impact of abdominal adiposity associated with generalized obesity, suggesting an emerging paradigm of abdominal adiposity. Moreover, overweight subjects had a non-significant finding, compared to obese subjects (as per the NIH classification) possibly because of abdominal adiposity, as all the obese class-II subjects and maximum $(83.87 \%)$ of obese class-I subjects had abdominal adiposity, while nearly half $(42.68 \%)$ of the overweight subjects had normal WHR, depicting the high prevalence of abdominal adiposity in obese subjects. However, present results do not confirm that adiposity is the primary factor underlying the differences between the subset of obese group of the present study.

The present study, however, represents a unique and practical effort with regard to the findings, suggesting that the fat distribution or visceral adiposity/abdominal adiposity (assessed by WHR) should be incorporated, together with BMI, in the routine clinical measurements of all the subjects to facilitate early identification, evaluation and treatment of obesity associated problems in the population.

\section{CONCLUSION}

Thus, it can be concluded that increased expression of 


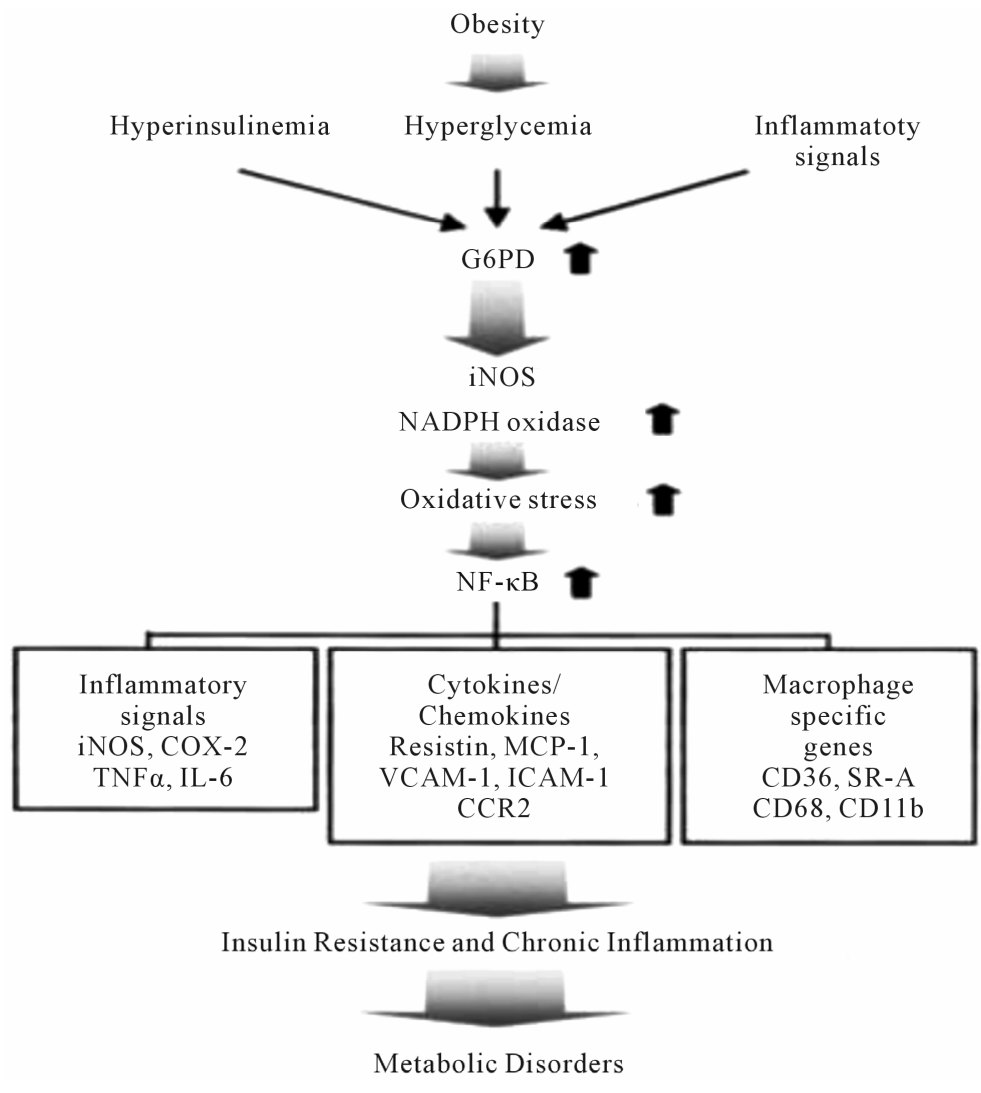

Figure 3. A model of G6PD-induced insulin resistance and chronic inflammation in adipocytes. Obesity-induced G6PD overexpression stimulates oxidative stress by increasing the expression of pro-oxidative enzymes, which are closely linked with NF- $\mathrm{kB}$ signals and their target gene expression, including cytokines, inflammatory signals, and macrophage-specific genes. These signals lead to metabolic disorders such as insulin resistance and chronic inflammation. The upward arrows indicate the increase of G6PD, iNOS, NADPH oxidase, oxidative stress, or NF- $\kappa$ B signaling. ICAM-1, intercellular adhesion molecule-1; VCAM-1, vascular cell adhesion molecule-1; SR-A, Scavenger receptor type A; CCR2, Chemokine receptor-2; COX-2, Cyclooxygenase-2; CD, Cluster of Differentiation; NF- $\kappa B$, Nuclear factor- $\kappa B$.

G6PD (a crucial enzyme in the maintenance of cellular redox potential) in obese subjects (more if it is associated with abdominal adiposity) might mediate the onset of obesity associated metabolic disorders by increasing oxidative stress.

Further, we propose that G6PD expression levels in adipose tissue may be a promising indicator of obesity associated metabolic syndrome and its regulation may be a novel therapeutic target in treating metabolic disorders, and therefore, the identification of regulatory tools for G6PD activity may prove promising for treating metabolic disorders such as obesity associated insulin resistance and chronic inflammation.

\section{REFERENCES}

[1] Zimmet, P., Alberti, K.G. and Shaw, J. (2001) Global and societal implications of the diabetes epidemic. Nature, 414, 782-787. doi: $10.1038 / 414782 \mathrm{a}$

[2] Nandeesha, Sathiyapriya, V., Bobby, Z., Selvaraj, N., Pavithran, P. and Agarawal, A. (2008) Atherogenic lipid risk factors in men classified as overweight and obese according to the preliminary WHO guidelines for Asians. Indian Journal of Physiology and Pharmacology, 52, 205-208.

[3] Grundy, S.M., Brewer Jr., H.B., Cleeman, J.I., Smith Jr., S.C. and Lenfant, C. (2004) Definition of metabolic syndrome: Report of the National Heart, Lung and Blood Institute/American Heart Association, Conference on scientific issue related to definition. Circulation, 109, 433-438. doi:10.1161/01.CIR.0000111245.75752.C6

[4] Spiegelman, B.M. and Filer, J.S. (2001) Obesity and the regulation of energy balance. Cell, 104, 531-543. doi:10.1016/S0092-8674(01)00240-9

[5] Montague, C.T. and O' Rahilly, S. (2000) The perils of 
portliness: Causes and consequences of visceral adiposity. Diabetes, 49, 883-888. doi:10.2337/diabetes.49.6.883

[6] Saltiel, A.R. and Kahn, C.R. (2001) Insulin signaling and the regulation of glucose and lipid metabolism. Nature, 414, 799-806. doi:10.1038/414799a

[7] Dandona, P., Aliada, A. and Bandyopadhay, A. (2004) Inflammation-the link between insulin resistance, obesity and diabetes. Trends in Immunology, 25, 4-7. doi:10.1016/j.it.2003.10.013

[8] Patel, D.A., Srinivasan, S.R., Xu, J.H., Chen, W. and Berenson, G.S. (2006) Adiponectin and its correlates of cardiovascular risk in young adults: The Bogalusa Heart Study. Metabolism, 55, 1551-1557. doi:10.1016/j.metabol.2006.06.028

[9] Perticone, F., Ceravolo, R., Candigliota, M., Ventura, G., Iacopino, S., Sinopoli, F. and Mattioli, P.L. (2001) Obesity and body fat distribution induce endothelial dysfunction by oxidative stress: Protective effect of Vitamin C. Diabetes, 50, 159-165. doi:10.2337/diabetes.50.1.159

[10] Finkel, T. and Holbrook, N.J. (2000) Oxidants, oxidative stress and biology of ageing. Nature, 408, 239-247. doi: $10.1038 / 35041687$

[11] Perreault, M. and Marette, A. (2001) Targeted disruption of inducible nitric oxide synthase protects against obesity-linked insulin resistance in muscle. Nature Medicine, 7, 1138-1143. doi:10.1038/nm1001-1138

[12] Furukawal, S., Fujita, T., Shimabukuro, M., Iwaki, M., Yamada, Y., Nakajima, Y., Nakayama, O., Makishima, M., Matsuda, M. and Shimomura, I. (2004) Increased oxidative stress in obesity and its impact on metabolic syndrome. Journal of Clinical Investigation, 114, 1752-1761.

[13] Salati, L.M. and Amir-Ahmady, B. (2001) Dietary regulation of expression of Glucose-6-Phosphate Dehydrogenase. Annual Review of Nutrition, 21, 121-140. doi:10.1146/annurev.nutr.21.1.121

[14] Horton, J.D., Goldstein, J.L. and Brown, M.S. (2002) SREBP $_{S}$ : Activators of the complete program of cholesterol and fatty acid synthesis in the liver. Journal of Clinical Investigation, 109, 1125-1131.

[15] Kletzien, R.F., Harris, P.K. and Foellmi, L.A. (1994) Glucose-6-phosphate dehydrogenase: A "house-keeping" enzyme subject to tissue specific regulation by hormones, nutrients and oxidant stress. FASEB Journal, 8, 174-181.

[16] Trinder, P. (1969) Determination of glucose in blood using glucose oxidase with an alternative oxygen acceptor. Annals of Clinical Biochemistry, 6, 24.

[17] Buccolo, G. and David, H. (1973) Quantitative determination of serum triglycerides by the use of enzymes. Clinical Chemistry, 19, 476-482.

[18] Allain, C.C., Poon, L.S., Chan, C.S.G., Richmond, W. and Fu, P.C. (1974) Enzymatic determination of total serum cholesterol. Clinical Chemistry, 20, 470-475.

[19] Assman, G., Schriewer, H., Schmitz, G. and Hagele, E.O. (1983) Quantification of high density lipoprotein cholesterol by precipitation with phosphotungstic acid/ $\mathrm{MgCl}_{2}$. Clinical Chemistry, 29, 2026-2030.

[20] Friedwald, W.T., Levy, R.I. and Fredrickson, D.S. (1972)
Estimation of the concentration of low-density lipoprotein cholesterol in plasma without use of the preparative ultracentrifuge. Clinical Chemistry, 18, 499-502.

[21] Satoh, K. (1978) Serum lipid peroxide in cerebrovascular disorders determined by a new colorimetric method. Clinica Chimica Acta, 90, 37-43. doi:10.1016/0009-8981(78)90081-5

[22] Makarem, A., et al. (1974) Clinical chemistry. Principles and techniques. In: Henry, R.F., Cannon, D.C. and Winelman, J.W., Eds., 2nd Edition, Harper and Row, Hagerstown, 1128-1135.

[23] National Institutes of Health, National Heart Lung and Blood Institute (1998) Clinical guidelines on the identification, evaluation and treatment of overweight and obesity in adults. Obesity Research, 6, 51S-210S. doi:10.1002/j.1550-8528.1998.tb00690.x

[24] WHO Report (2008) Waist circumference and waist-hip ratio. Report of a WHO Expert Consultation, WHO, Geneva.

[25] Silvio, E.I. (2010) Diabetes facts and guidelines, repot from American Diabetes Association. Diabetes Care, 33, S62.

[26] Executive summary of the third report of the National Cholesterol Education Program (NCEP) (2001) Expert panel on detection, evaluation and treatment of high blood cholesterol in adults (ATP III). JAMA, 285, 24862497.

[27] Beutler, E. and Vulliamy, T.J. (2002) Hematologically important mutations: Glucose-6-phosphate dehydrogenase. Blood Cells, Molecules and Diseases, 28, 93-103. doi:10.1006/bcmd.2002.0490

[28] Dessi, S., Batetta, B., Spano, O., Pulisci, D., Mulas, M.F., Muntoni, S., Armeni, M., Sanna, C., Antonucci, R. and Pani, P. (1992) Serum lipoprotein pattern as modified in G6PD-deficient children during hemolytic anemia induced by fava bean ingestion. International Journal of Experimental Pathology, 73, 157-160.

[29] Carvalho-Filho, M.A., Ueno, M., Hirabara, S.M., Seabra, A.B., Carvalheira, J.B., De Oliveira, M.G., Velloso, L.A., Curi, R. and Saad, M.J. (2005) S-nitrosation of the insulin receptor, insulin receptor substrate 1 , and protein kinase b/akt: A novel mechanism of insulin resistance. Diabetes, 54, 959-967. doi:10.2337/diabetes.54.4.959

[30] Yasukawa, T., Tokunaga, E., Ota, H., Sugita, H., Martyn, J.A. and Kaneki, M. (2005) S-nitrosylation dependent inactivation of $\mathrm{AKt} /$ protein kinase $\mathrm{B}$ in insulin resistance. Journal of Biological Chemistry, 280, 7511-7518. doi:10.1074/jbc.M411871200

[31] Park, J., Rho, H.K., Kim, K.H., Choe, S., Lee, Y.S. and Kim, J.B. (2005) Overexpression of Glucose-6-phosphate dehydrogenase is associated with lipid dysregulation and insulin resistance. Molecular and Cellular Biology, 25, 5146-5157. doi:10.1128/MCB.25.12.5146-5157.2005

[32] Park, J., Choe, S.S., Choi, A.H., Kim, K.H., Yoon, M.J., Suganami, T., Ogawa, Y. and Kim, J.B. (2006) Increase in Glucose-6-phosphate dehydrogenase in adipcyte stimulates oxidative stress and inflammatory signals. Diabetes, 55, 2939-2949. doi: $10.2337 / \mathrm{db} 05-1570$ 
[33] Arkan, M.C., Hevener, A.L., Greten, F.R., Maeda, S., Li, Z.W., Long, J.M., Wynshaw-Boris, A., Poli, G., Olefsky, J. and Karin, M. (2005) IKK-beta links inflammation to obesity-induced insulin resistance. Nature Medicine, 11, 191-198. doi: $10.1038 / \mathrm{nm} 1185$
[34] Cai, D., Yuan, M., Frantz, D.F., Melendez, P.A., Hansen, L., Lee, J. and Shoelson, S.E. (2005) Local and systemic insulin resistance resulting from hepatic activation of IKK-beta and NF-Kappa B. Nature Medicine, 11, 183190. doi: $10.1038 / \mathrm{nm} 1166$ 